\title{
Transient scattering effects and electron plasma dynamics during ultrafast laser ablation of water
}

\author{
Javier Hernandez-Rueda* and Dries van Oosten \\ Debye Institute for Nanomaterials Science and Center for Extreme Matter and Emergent Phenomena, Utrecht University, \\ Princetonplein 5, 3584 CC Utrecht, Netherlands \\ *Corresponding author: fjavihr@gmail.com
}

Received 11 January 2019; revised 7 March 2019; accepted 8 March 2019; posted 8 March 2019 (Doc. ID 357531); published 1 April 2019

We study the dynamics of single-shot ultrafast laser ablation of a water-gas interface. We model the extremely nonlinear light-water interaction during the first picosecond by simulating the laser pulse propagation while dynamically calculating the spatial distribution of the dielectric function. We make use of a finite-difference time-domain algorithm to solve Maxwell's equations and Rethfeld's multiple rate equation model to consider the local excitation of a dense electron plasma. We validate our model by comparing the simulated transient reflectivity with experimental results and find excellent agreement. (๑) 2019 Optical Society of America

https://doi.org/10.1364/OL.44.001856

Provided under the terms of the OSA Open Access Publishing Agreement

Pulsed lasers are used in a wide variety of fields in research, technology, and life science [1-7]. The interaction of laser pulses with aqueous targets, which is especially relevant in laser-based cell surgery and clinical surgery $[8,9]$, has been studied using femtosecond ( $\mathrm{fs}$ ), picosecond (ps), and nanosecond (ns) pulse durations. For fs laser ablation, the physics is particularly appealing as it consists of a chain of mechanisms that are well separated in time $[7,10,11-14]$, due to the ultrafast energy deposition and the nonlinearity of the process [15]. During the initial strong nonlinear interaction of the fs laser pulse and the water, a dense and hot electron plasma is created $[10,16,17]$. On a picosecond time scale, the electrons and molecules thermalize, bringing the water to an extremely superheated phase. On a ns time scale, this leads to a violent supersonic expansion of the water vapor, which in turn drives the formation of a shockwave in the surrounding medium. The dynamics of this process have experimentally been studied using time-resolved shadowgraphy and time-resolved microscopy in reflection mode, which provide extensive information on the expansion dynamics of the ps-ns- $\mu$ s time regimes $[1,2,7,10,18-24]$. The initial laser-matter interaction and the formation of freeelectron plasma have been theoretically studied using rateequation-based models $[10,11,17]$. However, the effect of the dynamically changing optical properties on the propagation of the laser pulse into the target must be taken into account to accurately describe the energy deposition process.
In this Letter, we present a model of the laser excitation during ultrafast laser ablation at a water-gas interface. The model takes into account the laser pulse propagation and the extremely nonlinear interaction with water within a pumpand-probe-type system. To simulate the propagation, we implement a two-dimensional finite-difference time-domain (2D-FDTD) routine [3,25]. To model the light-water interaction, we consider the Drude model, the Kerr effect, the multiple rate equation (MRE) method [16,17,26], and the Keldysh strong-field ionization formula $[27,28]$. We compare the results of the model with experimental data acquired using pump $\left(\lambda_{\mathrm{IR}}=800 \mathrm{~nm}\right)$ and probe $\left(\lambda_{\mathrm{UV}}=400 \mathrm{~nm}\right)$ timeresolved microscopy recently reported by our group [23,24]. In Fig. 1, we reproduce typical transient reflectivity images from that work at relevant time delays immediately after a single laser pulse exposure at a water surface. We attribute the surface reflectivity increase to the excitation of a free-electron plasma.

During the simulation, we compute the electron plasma density using the MRE method in order to take the light coupling to the free electrons and the process of avalanche ionization into account. This model considers a distribution of free electrons in effective discrete energy levels, whose spacing corresponds to the laser photon energy $\hbar \omega=1.56 \mathrm{eV}[16,17,26]$. Free electrons can gain energy via single-photon excitation and ionize bound electrons via impact ionization when their energy exceeds the effective ionization potential [26]. This method is summarized by the equations
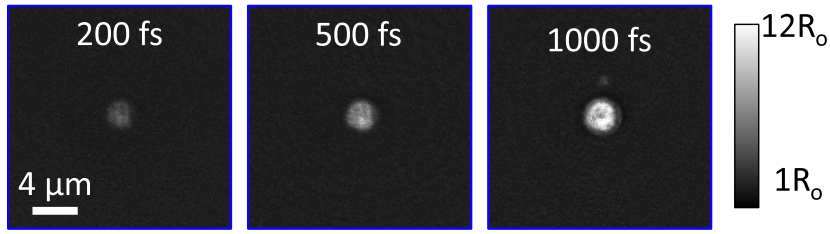

Fig. 1. Transient relative reflectivity images of the fs-laser-excited water surface during the first picosecond, previously reported in Ref. [23]. $R_{o}$ corresponds to the reflectivity for an unexcited waterair interface. The images share the same lateral scale $\left(20 \times 20 \mu \mathrm{m}^{2}\right)$ and are represented using the same color scale. 


$$
\begin{aligned}
\dot{\rho}_{0} & =-W_{1 \mathrm{pt}} \rho_{0}+W_{\mathrm{SFI}}+2 \alpha \rho_{k}, \\
\dot{\rho}_{1} & =-W_{1 \mathrm{pt}} \rho_{1}+W_{1 \mathrm{pt}} \rho_{0}, \\
& \vdots \\
\dot{\rho}_{k-1} & =-W_{1 \mathrm{pt}} * \rho_{k-1}+W_{1 \mathrm{pt}} \rho_{k-2}, \\
\dot{\rho}_{k} & =-\alpha \rho_{k}+W_{1 \mathrm{pt}} \rho_{k-1},
\end{aligned}
$$

where $k=\left\lceil U_{\text {eff }} / \hbar \omega\right\rceil$ is the number of effective energy levels, $\alpha\left(1 \mathrm{fs}^{-1}\right)$ is the avalanche ionization coefficient [16,11], and $\mathrm{W}_{1 \mathrm{pt}}$ denotes the one-photon absorption coefficient $[16,29]$. We do not take thermal or electron diffusion into account. We compute the strong-field ionization rate $\mathrm{W}_{\mathrm{SFI}}$ (i.e., multiphoton and tunnel ionization $[6,27]$ ) as a function of the electric field strength $\mathrm{E}(\boldsymbol{r}, t)$ at $800 \mathrm{~nm}$, considering an ionization potential for unexcited water of $U_{0}=9.5 \mathrm{eV}$ [10]. Following Christenseen and Balling [26], we estimate the scattering rate $\Gamma_{e-e}$, the electron temperature $T$, and density $\rho$ using

$$
\begin{gathered}
k_{B} T=\frac{2}{3} \frac{\sum_{i=0}^{k} i \hbar \omega \rho_{i}}{\sum_{i=0}^{k} \rho_{i}}, \quad \rho(\boldsymbol{r}, t)=\sum_{i=0}^{k} \rho_{i}(\boldsymbol{r}, t), \\
\Gamma_{e-e}(\boldsymbol{r}, t)=\frac{4 \pi \epsilon_{0}}{e^{2}} \sqrt{\frac{6}{m_{e}}}\left(k_{B} T\right)^{3 / 2}, \\
\Gamma(\boldsymbol{r}, t)=\Gamma_{e-p}(\boldsymbol{r}, t)+\Gamma_{e-e}(\boldsymbol{r}, t),
\end{gathered}
$$

where $\epsilon_{0}$ is the permittivity of free space, $k_{B}$ is the Boltzmann constant, $e$ and $m_{e}$ are the charge and mass of the electron, $\omega$ is the frequency of the laser, and $\rho_{i}$ is the spatial and temporal distributions of electrons in the $i$ th level.

The dielectric function is calculated considering Drude and Kerr contributions as follows:

$$
\epsilon(\boldsymbol{r}, t)=\epsilon_{\mathrm{water}}-\frac{\omega_{p}(\boldsymbol{r}, t)^{2}}{\omega^{2}+i \omega \Gamma(\boldsymbol{r}, t)}+\frac{3}{4} \chi_{3}|E(\boldsymbol{r}, t)|^{2},
$$

where $\chi_{3}$ is the third-order nonlinear susceptibility and $\omega_{p}(\boldsymbol{r}, t)^{2}=e^{2} \rho(\boldsymbol{r}, t) / m_{e} \epsilon_{0}$ is the plasma frequency that depends on the time- and position-dependent electron plasma density, which follows from Eq. (2), and is therefore updated dynamically within the FDTD algorithm. Table 1 presents the optical properties of water used during the simulation. Note that all model parameters are taken from experimental studies in the literature.

Figure 2(a) shows a block diagram of one cycle of the FDTD routine for both pump and probe pulses. We solve Maxwell's equations within the simulation box and extract both the electric and magnetic fields. More details regarding our FDTD routine can be found in Refs. [3,25]. Every optical half-cycle

Table 1. Water Properties Used in the Simulations

\begin{tabular}{lcc}
\hline Parameter & Value & Source \\
\hline Refractive index (IR) & $1.329+i 1.2510^{-7}$ & {$[30]$} \\
Refractive index (UV) & $1.336+i 1.8610^{-9}$ & {$[30]$} \\
$n_{2}$ & $1.8610^{-16} \mathrm{~cm}^{2} / \mathrm{W}$ & {$[1]$} \\
Effective mass pump & $0.5 * m_{e}$ & {$[16]$} \\
Effective mass probe & $0.4 * m_{e}$ & {$[10]$} \\
$\Gamma_{e-p}$ & $1 \mathrm{fs}^{-1}$ & {$[11]$} \\
\hline
\end{tabular}

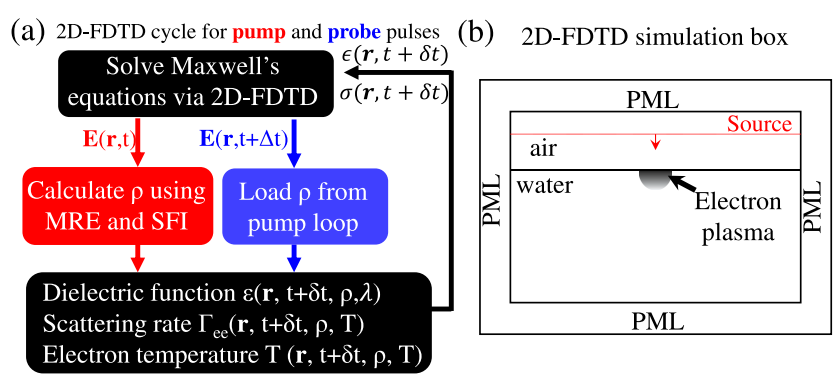

Fig. 2. (a) Block diagram of the simulation procedure that combines 2D-FDTD with MRE for a pump (red) and probe (blue) cycles. (b) 2D-FDTD simulation box. Scattered and backreflected $E$ and $H$ values are stored within the whole simulation grid to extract the transient reflectivity and illustrate the transient scattering effects.

(i.e., $\delta t$ ), we combine the spatial distribution of the electric field strength $E(r, t)$ with Eqs. (1)-(3) to compute the electron plasma density, temperature, and scattering rate. These are then used to retrieve the dielectric function $\epsilon(r, t+\delta t)$ and plasma conductivity $\sigma(r, t+\delta t)$ that will be used during the next optical half-cycle. During the simulation of the propagation of the pump beam [red block in Fig. 2(a)], $\epsilon(r, t)$ and $\sigma(r, t)$ are also stored to file. Subsequently, the simulation of the probe beam is done for different time delays $\Delta t$, by loading the transient material properties stored during the pump simulation that aid to calculate $\epsilon(r, t+\Delta t)$ and $\sigma(r, t+\Delta t)$ [blue block in Fig. 2(a)]. We assume the probe does not influence the optical properties as its fluence is $10^{4}$ times lower than that one of the pump. Figure 2(b) presents the FDTD simulation box that has a height $(z)$ of $5 \mu \mathrm{m}$ and a width $(r)$ of $14 \mu \mathrm{m}$ with a grid spacing of $35 \mathrm{~nm}$. The perfectly matched layer (PML) consists of 60 grid points that minimize reflections at the boundaries of the simulation space. The total simulation time extends over approximately five times the pulse duration of the laser pulse ( $\approx 800 \mathrm{fs})$. We use spatial and temporal Gaussian distributions for the electric field sources. To set the features of the pump pulse in the simulation, we use the actual conditions employed during the experiments reproduced in Fig. 1, i.e., a duration of $150 \mathrm{fs}$ (full width half-maximum), a wavelength of $800 \mathrm{~nm}$, a fluence of $25 \mathrm{~J} / \mathrm{cm}^{2}$, and a beam waist of $3.4 \mu \mathrm{m}\left(1 / e^{2}\right)$. The probe pulse fluence was measured to be $2 \mathrm{~mJ} / \mathrm{cm}^{2}$, which is neglected in the excitation of the electron plasma. We use the duration of the probe pulse as the only free parameter during the simulations. We find a value of $400 \mathrm{fs}$ that is consistent with our estimate after passing through the objective and several lenses (i.e., $325 \mathrm{fs}$ ). The transient reflectivity is obtained by retrieving the time-integrated Poynting vector of the backreflected probe beam. To benchmark the reflectivity simulations using our 2D-FDTD code, the reflectivity of the probe beam for unexcited water and gold were obtained and compared with the ones calculated using the Fresnel formula, showing a relative error below 5\% [3,25].

In Figs. 3(a) and 3(b), we show 2D $(r-z)$ false color maps of the calculated electric field strength of the pump pulse and the spatial distribution of the electron plasma density, respectively. Note that the laser pulse impinges from the top of the $2 \mathrm{D}$ maps at the water-air interface $(z=0)$ and propagates downward inside the water sample along the $z$ axis. These maps illustrate that the electron plasma drastically modifies 
(a)
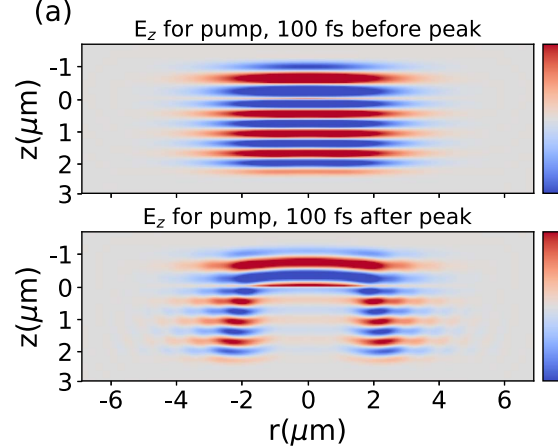

(b)

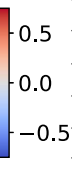

Electron density, $100 \mathrm{fs}$ before pump peak

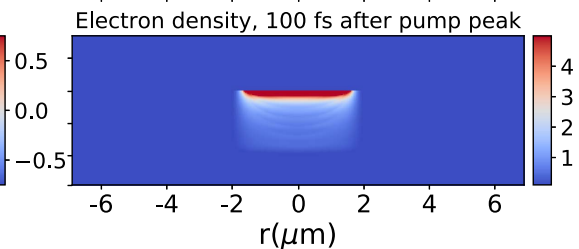

(c)

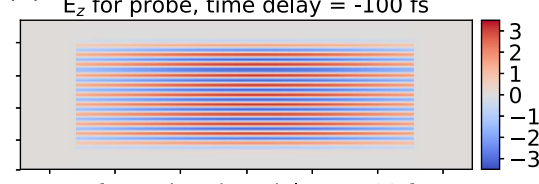

$E_{z}$ for probe, time delay $=700 \mathrm{fs}$

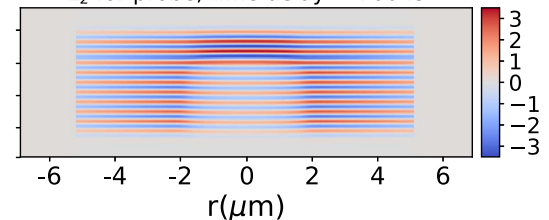

Fig. 3. (a) Simulated electric field of the pump beam $\left(10^{10} \mathrm{~V} / \mathrm{m}\right)$ and (b) the corresponding electron plasma density maps $\left(10^{20} \mathrm{~cm}^{-3}\right)$ achieved $100 \mathrm{fs}$ before (top row) and after (bottom row) the laser peak intensity. (c) Simulated electric field of the probe beam $\left(10^{8} \mathrm{~V} / \mathrm{m}\right)$ at $-100 \mathrm{fs}$ and $700 \mathrm{fs}$ time delays.

the transient optical properties and strongly influences the manner in which the remaining fraction of the pump pulse reflects and scatters, as our group similarly found for silicon on insulator targets [25]. This effect is illustrated in the maps of the electric field achieved 100 fs before (top row, $n_{e}^{\max }=1.2 \cdot 10^{20} \mathrm{~cm}^{-3}$ ) and after (bottom row, $n_{e}^{\max }=$ $8 \cdot 10^{21} \mathrm{~cm}^{-3}$ ) the laser peak intensity. Figure $3(\mathrm{c})$ presents the results of the probe simulations. We show the maps of the electric field (centered at the peak intensity of the probe) of two probe pulses at $-100 \mathrm{fs}$ and $700 \mathrm{fs}$ time delays. We observe that the probe field is strongly scattered and reflected for a longer delay (i.e., $700 \mathrm{fs}$ ) by the highly dense electron plasma $\left(n_{e}^{\max }=1.2 \cdot 10^{22} \mathrm{~cm}^{-3}\right)$. We verify our simulation by comparing the simulated transient relative reflectivity at short time delays $(<1 \mathrm{ps})$ to experimental data from [23]. The solid lines in Fig. 4 show the results of the reflectivity simulations obtained by calculating the time-integrated Poynting vectors for the probe pulse at several pump-probe delays. The colored circles illustrate azimuthal averages of the transient reflectivity retrieved from the experimental time-resolved microscopy images. We find good correspondence between

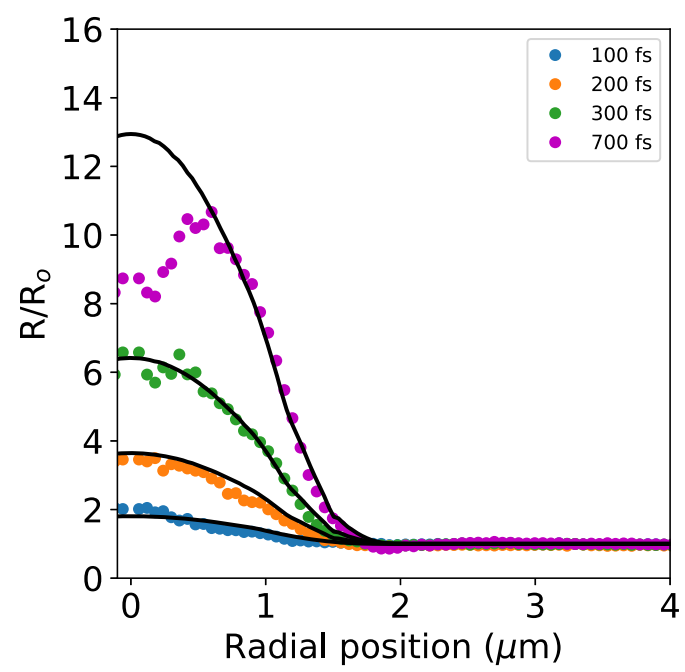

Fig. 4. Experimental (markers) and simulated (solid lines) transient reflectivity for several time delays. The experimental data were reported by our group in Ref. [23]. the experiment and the model, which validates the pump pulse simulation. In particular, we observe excellent agreement for short delays. At 700 fs time delay, we observe an obvious mismatch, which we attribute to the fact that our model does not take into account any hydrodynamics and therefore does not describe the reduced reflectivity that can arise from the expanding vapor.

Figure 5(a) shows the time dependence of the maximum electron plasma density and temperature of the irradiated volume, reaching values of $1.2 \cdot 10^{22} \mathrm{~cm}^{-3}$ and $28,000 \mathrm{~K}$ at $700 \mathrm{fs}$. It is worth noting that the maximum temperature is achieved at $450 \mathrm{fs}$, after which the simulation shows a decrease
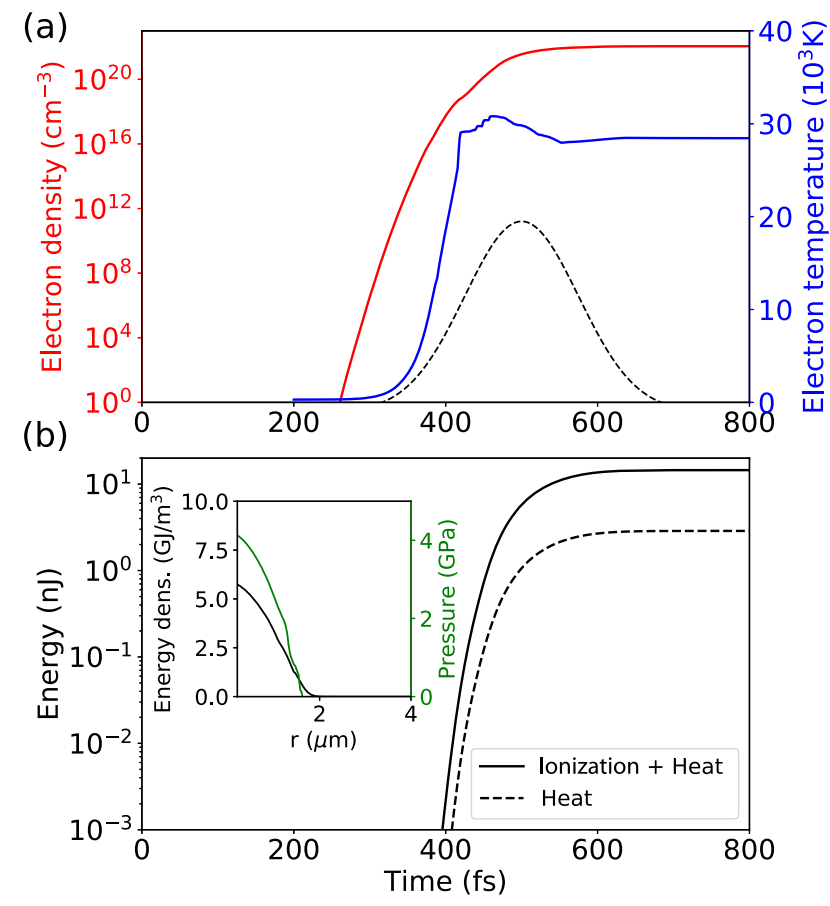

Fig. 5. Graphs of the (a) electron plasma density and temperature and (b) total and thermal energies as a function of time. The dashed line in (a) shows the intensity profile of the laser pulse. The inset in (b) shows the radial dependence of the energy density (just below the surface) and the local pressure obtained after subtracting the energy required to overcome the enthalpy of evaporation. 
of the electron plasma temperature by $2000-3000 \mathrm{~K}$ (depending on the spatial position). This is due to the effect of avalanche ionization in which heat of the electron plasma is used to create more free electrons. The simulation provides the spatial distribution of the electron density and temperature as well as the free-electron kinetic energy distribution and scattering time. From these maps, we can also calculate how much of the absorbed laser energy is used to excite bound electrons and how much is used to heat the resulting electron plasma. We find that, with a fluence of $25 \mathrm{~J} / \mathrm{cm}^{2}$ and a beam waist of $3.4 \mu \mathrm{m}$, an energy of $14 \mathrm{~nJ}$ is absorbed, of which $4 \mathrm{~nJ}$ is in the form of heat of the electron plasma as shown in Fig. 5(b). Furthermore, the radial dependence of the kinetic energy density at the end of the simulation is shown in the inset within Fig. 5(b).

By comparing the final energy density distribution to the specific energy required to heat and evaporate water, we can estimate the volume of water that can be evaporated, assuming the evaporation process is fast enough so that thermal diffusion can be neglected. We find a disk-like volume with a radius of $\sim 1.50 \mu \mathrm{m}$ and a depth of $\sim 70 \mathrm{~nm}$, which corresponds to $5 \cdot 10^{-16} \mathrm{~kg}$ of water. Given the extremely small depth, we have to verify diffusion can in fact be neglected. From the maximum electron temperature reached during the simulation, we estimate an upper limit of $\sim 30 \mathrm{~nm}$ for the thermal diffusion length on the time scale of 1 ps. From this, we see that it is reasonable to neglect the effect of thermal diffusion in this regime. For higher fluences, the MREs should be extended to include diffusion.

The available heat after evaporation is sufficient to heat the water to a maximum temperature of several thousand Kelvin. The thermodynamic state of the system is well beyond the spinodal limit and thus extremely superheated. Due to the lack of an appropriate equation of state for such extreme and dynamically changing supercritical conditions [31], we use the equation of the state of an ideal gas to make an estimate of the pressure distribution. This seems a reasonable approximation as the energy per water molecule significantly exceeds the hydrogen bond strength. The resulting local pressure distribution after laser excitation is shown in Fig. 5 (b) (green line), reaching values of $4.2 \mathrm{GPa}$. For comparison, we can compute the peak pressure using a much more sophisticated equation of state [32], which yields a peak pressure of $23.5 \mathrm{GPa}$. The thermodynamic state of the water vapor at the end of the 2D-FDTD simulation can serve as the initial condition to model the expansion of the system using computational fluid dynamics.

In conclusion, we theoretically investigate the ultrafast interaction of a single fs laser pulse with water during the first ps. We combine a FDTD routine with the MRE method to simultaneously account for the laser pulse propagation and its interaction with the water target. In this way, we calculate the time dependence of the spatial distribution of the dielectric function that is mainly influenced by laser-induced free-electron plasma density, temperature, and scattering time. We observe transient scattering effects on the electric field of the pump pulse when it interacts with the micron-sized electron plasma, which reaches a maximum density of $10^{22} \mathrm{~cm}^{-3}$ and a temperature of 28,000 K. Our model excellently reproduces the dynamic behavior of experimentally measured reflectivity and gives an estimate of the thermodynamic state of the water during and after laser excitation.

Funding. H2020 Marie Skłodowska-Curie Actions (MSCA) (703696 ADMEP).

Acknowledgment. We thank Denise M. Krol for fruitful discussions. We also thank Paul Jurrius, Cees de Kok, and Dante Killian for technical assistance.

\section{REFERENCES}

1. C. B. Schaffer, N. Nishimura, E. N. Glezer, A. M.-T. Kim, and E. Mazur, Opt. Express 10, 196 (2002).

2. B. Strycker, M. Springer, A. Traverso, A. Kolomenskii, G. Kattawar, and A. Sokolov, Opt. Express 21, 23772 (2013).

3. H. Zhang, S. Wolbers, D. Krol, J. Dijkhuis, and D. Van Oosten, J. Opt. Soc. Am. B 32, 606 (2015).

4. R. R. Gattass and E. Mazur, Nat. Photonics 2, 219 (2008).

5. U. K. Tirlapur and K. König, Nature 418, 290 (2002).

6. J. Hernandez-Rueda, J. Clarijs, D. van Oosten, and D. M. Krol, Appl. Phys. Lett. 110, 161109 (2017).

7. A. Vogel and V. Venugopalan, Chem. Rev. 103, 577 (2003).

8. M. F. Yanik, H. Cinar, H. N. Cinar, A. D. Chisholm, Y. Jin, and A. Ben-Yakar, Nature 432, 822 (2004).

9. S. X. Guo, F. Bourgeois, T. Chokshi, N. J. Durr, M. A. Hilliard, N. Chronis, and A. Ben-Yakar, Nat. Methods 5, 531 (2008).

10. N. Linz, S. Freidank, X.-X. Liang, H. Vogelmann, T. Trickl, and A. Vogel, Phys. Rev. B 91, 134114 (2015).

11. T. Winkler, C. Sarpe, N. Jelzow, L. Haahr-Lillevang, N. Götte, B. Zielinski, P. Balling, A. Senftleben, and T. Baumert, Appl. Surf. Sci. 374, 235 (2016).

12. C. Sarpe, J. Köhler, T. Winkler, M. Wollenhaupt, and T. Baumert, New J. Phys. 14, 075021 (2012).

13. F. Courvoisier, V. Boutou, C. Favre, S. C. Hill, and J.-P. Wolf, Opt. Lett. 28, 206 (2003).

14. C. Sarpe-Tudoran, A. Assion, M. Wollenhaupt, M. Winter, and T. Baumert, Appl. Phys. Lett. 88, 261109 (2006).

15. D. von der Linde, K. Sokolowski-Tinten, and J. Bialkowski, Appl. Surf. Sci. 109-110, 1 (1997).

16. B. Rethfeld, Phys. Rev. Lett. 92, 187401 (2004).

17. B. Rethfeld, Phys. Rev. B 73, 035101 (2006).

18. I. Apitz and A. Vogel, Appl. Phys. A 81, 329 (2005).

19. T. T. Nguyen, R. Tanabe, and Y. Ito, Opt. Laser Technol. 100, 21 (2018).

20. Z. I. Nagy, A. Takács, T. Filkorn, and M. A. Sarayba, J. Refractive Surg. 2009, 25 (2009).

21. R. R. Krueger, J. S. Krasinski, C. Radzewicz, K. G. Stonecipher, and J. J. Rowsey, Cornea 12, 330 (1993).

22. T. Juhasz, X. H. Hu, L. Turi, and Z. Bor, Lasers Surg. Med. 15, 91 (1994).

23. J. Hernandez-Rueda and D. van Oosten, "Dynamics of ultrafast laser ablation of water," arXiv:1810.06946 (2018).

24. M. Vreugdenhil, D. van Oosten, and J. Hernandez-Rueda, Opt. Lett. 43, 4899 (2018).

25. H. Zhang, D. M. Krol, J. I. Dijkhuis, and D. van Oosten, Opt. Lett. 38, 5032 (2013).

26. B. H. Christensen and P. Balling, Phys. Rev. B 79, 155424 (2009).

27. V. Gruzdev, Phys. Rev. B 75, 205106 (2007).

28. L. Keldysh, Sov. Phys. JETP 20, 1307 (1965).

29. L. Englert, B. Rethfeld, L. Haag, M. Wollenhaupt, C. Sarpe-Tudoran, and T. Baumert, Opt. Express 15, 17855 (2007).

30. M. Polyanskiy, 2014, http://refractiveindex.info.

31. D. Pan, Q. Wan, and G. Galli, Nat. Commun. 5, 3919 (2014).

32. W. Wagner and A. Pruß, J. Phys. Chem. Ref. Data 31, 387 (2002). 